\title{
Variables affecting fans' loyalty to Egyptian football clubs' brands
}

\section{Introduction and research problem}

*Dr/ Ahmed El Sayed Ali Al Hosiny

Brand loyalty is one of the topics that occupy a large part of marketers' interest in general and sports marketers in particular, where sports clubs seek to enhance loyalty of current consumers within commercialization era of football industry especially with the active role played by consumers in this industry. So, clubs make efforts to preserve current consumers and also attract new ones, which is reflected on brand value, that ultimately translates into financial revenues represented in: viewing rates, match tickets, commercial sales and sports sponsorship.

Football brands are unique, whether they are: clubs, players, or the game itself. There is a level of participation among football fans that makes them hungry to understand clubs and the game. This is what makes extraordinary loyalty of football fans clearly visible in difficult times. And, this is what makes football brands characterized with uniqueness. (7: 173)

It is important to note that there is an emotional connection between fans and football brands, and it is normal that individuals' feelings and moods to be affected by their team loss or success (10:309). This indicates that, the more people become connected with any team, the more team value increases to those people, the greater their willingness to participate in its activities. (6:502)

This association with brand in football industry results in a different behavior than other types of industries. If a consumer had a bad experience with a brand in an industry, his response will be changing to another brand, so marketers work on "preserving consumers" in loyalty circle to their brand, although it is

" Lecturer at Sport Management Department, Faculty of Physical Education, Mansoura University Assiut Journal For Sport Science Arts 
recognized that both high consumer expectations and competition can result in changing brand. (7:177)

In sports, we find that response from football fans is stronger than it is in any other industry. Fans derive strength and pride from their affiliations with any team; they often see their team as an extension of themselves, meaning that team success is a personal success and team failure is a personal failure. (15:182)

(Biscaia, $\mathrm{R}$ et al 2015) point according to (McDonald, \& Funk) Today, sports teams are seen as brands in themselves, where the brand considers the most important asset of sports teams. In sports context, brand's value is determined by its position between fans. The idea of managing a team as a brand became a dominant model in sports market . (5: 159)

Brand Finance report (2011) confirms that brands is a large intangible asset, especially for successful and famous sporting entities. So, most successful football clubs need to recognize the need to understand brand value in order to make better strategic decisions. (9:1)
Within light of football greatness in our current society and values it represents for various categories of people in their daily lives, supporters of national federations and professional clubs who work in football industry have become loyal buyers of clubs' or federations' brands; either they were performing strongly or weakly. This is the essence of brand's loyalty in football industry, where loyalty transferred to all consumer merchandise bearing clubs' or federations' brands. (2:233)

Through literature review on brand loyalty, two concepts emerged: attitudinal loyalty, behavioral loyalty. In sports context, attitudinal loyalty consists of: knowledge of the club, club's importance in fans' life, fans' personal feeling of team's success and failure. Behavioral loyalty represented in: fans' frequent consumption, purchasing behaviors, for example: following games and buying club's merchandise. (14:49)

Over the past four decades, concept of loyalty has been explored on a very wide scale. Researchers found that loyal sports fans attend more 
matches and buy more clubs' branded products, and support the club to gain public funds. (4:215) Thus, professional sports clubs aim to identify and understand factors that cause fans' loyalty for reasons similar to those of commercial organizations (10:307).

Due to financial pressures facing clubs in general and increasing competition in market, sports marketers need to understand fans' perceptions of teams to develop marketing strategies aimed at increasing teams' brand value, and to engage fans in positive subsequent practices towards the team, such as: attending more games, purchasing other products or services (18:90)

One of the challenges facing sports marketers and managers is to understand and interpret underlying causes of loyalty towards football clubs' brands, to establish relationships with fans and develop strategies to take advantage of this phenomenon for the benefit of sports clubs. This study seeks to identify the most influential variables in loyalty phenomenon towards football clubs' brands to provide a knowledge structure for sports marketers and managers about these variables, which helps them to manage and consolidate strong and lasting relationships with fans of the club.

\section{Research terms:}

Fans: someone who has strong loyalty to a specific club or individuals within the club. (12:34)

Brand: Is a "name, word, sign, symbol, graphic, or combination of all that". Any brand serves the purpose of identifying merchandise and services for any company and distinguishing them from competitors. (15:180)

${ }^{1}$ Behavioral loyalty: sports' fan repeated use of branded products of a particular club such as watching televised games, attending matches, wearing team clothes.

\section{Literature review:}

- Bauer et al (2008), aimed to identifying impact of brand image on fans' loyalty in team sports, where data been collected through electronic survey on a sample consisted of (1298) fans of Bundesliga. The most important results were: there are relationships between brand image 
components, that affects positively on fans' loyalty towards brand, brand's attributes non related to products (such as logo or tradition) have a very strong impact on attitudes and behavior, and consider a starting points for brand's successful and distinctive strategy.

- $\quad$ Richelieu et al (2011), aimed to understanding how managers can build a successful football team's brand at different levels of competition. The researchers used case study method of seven Football teams (three from Germany - three from France - one from Spain). The most important results were that driving factors in building brand identity and loyalty are: club history, performance onfield, style of play, foreign stars, local stars, team managers, logo, public relations and community involvement, different management strategies for brands between teams in secondary and Champions League.

- Saad Ahmed Shalaby, Naji Ismail Hamed (2013), aimed to identifying differences between national federations and football clubs in Egypt and Emirates in managing licensing programs with using brands in football industry, total sample size was (40) marketing managers, the most important results: there are significant differences with regard to some marketing efforts and legal rules that must be included at managing brands' licensing agreements.

- Martin, C.A. (2013), aimed to determine whether consumer's motivation and commitment affect his loyalty to his favorite team in NFL. The study used survey responses from (302) NFL fans. The results indicated that increasing social motivation of NFL fans in consumption increases their commitment, in turn; increasing NFL fans' commitment increases their loyalty, which also affects their behavioral loyalty.

- Hoegele et al (2014), aimed to studying star players' influence on attracting football fans to competitions. The researchers used descriptive approach, study sample included (1814) from football fans in Bundesliga. The most important results were that star players attract new fans and Assiut Journal For Sport Science Arts 
contribute in preserving current fans.

- Dina Abdel Hakim (2015), aimed to assessing corporate sponsorship impact on global sporting events as a marketing tool. The study was applied to European Champions League 2013-2014 to measure corporate sponsorship impact on brand's value on Egyptian consumer for this event. The most important results were: agreement between research sample on importance of encouraging their preferred team and considering this team an integral part of their personal lives, where research sample preference for a player or more of those who participate in sports event affects loyalty to event's commercial sponsors.

- Biscaia et al (2015), aimed to study differences in perceptions about the brand's standing among club members and non-members Researches used brand's position scale; research sample included (2287) fans of Portuguese football league. The most important results were significant differences in: brand dimensions, social interaction, commitment, team history, organizational characteristics, team success, technical manager, management, and stadium. The results of SEM analysis showed that relationships between brand position, dimensions and behavioral intentions do not differ significantly between groups.

- Maderer et al. (2016), aimed to comparing impact of brand ties on fans' brand loyalty in DFM and EFM markets. Research sample total was (2032) fans from developed markets (Germany, England, Spain, Italy and France), as well as 1555 fans from emerging markets (Brazil, Russia, India, China and USA). The most important results were: negative impact of brand attributes on attitudinal loyalty, marketers should not apply same strategies that have proven effectiveness in developing brand loyalty at developed markets than fans in emerging markets.

- $\quad$ Kargaluoto et al (2016), aimed to test direct and indirect effects of any sports team personality, identification with team, impact of fans' relationship length any team and their loyalty to it. Researchers used descriptive 
approach and applied electronic survey to a sample of (1166). The most important results were: brand personality is a strong guide to identification among new fans. Results also confirmed on the importance of sports brand personality in fans' identification with the team and their loyalty to it.

\section{Research Goal:}

The aim of this research is to determine variables affecting fans' loyalty to Egyptian football clubs' brands and to arrange them by testing validity of following assumptions:

\section{Research hypotheses:}

1. There is a positive correlation between club's variables (club popularity, winning and performance onfield, players, club management) and loyalty to brand.

2. Club's variables (club's popularity, winning and performance on-field, players, club management) all influence on loyalty to brand.
3. There is a positive correlation between fan's variables (community pride, social aspects, and fan's identity) and loyalty to brand.

4. Fan's variables (community pride, social aspects, and fan's identity) all influence on loyalty to brand.

\section{Research procedures:}

\section{Research Methodology:}

The researcher used descriptive method (survey method). The research aimed to explain loyalty phenomenon to brands in football through determining their dimensions and describing this phenomenon's relations.

\section{Community and Search}

\section{Sample:}

Research community:

Research community is composed of Fans of Egyptian football clubs of all ages.

\section{Research sample:}

The researcher randomly selected research sample, that consisted (815) fans divided to (75) fans for exploratory sample and (740) fans for basic study. 
Table (1)

Numbers and percentages of basic sample according to basic data $\mathrm{n}=\mathbf{( 7 4 0 )}$

\begin{tabular}{c|l|c|c}
\hline \hline & Variables & Number & Percentage\% \\
\hline \hline \multirow{3}{*}{ Gender } & Male & 438 & $59.18 \%$ \\
\cline { 2 - 4 } & Female & 302 & $40.82 \%$ \\
\hline \multirow{4}{*}{ Age } & Less than 20 years & 147 & $19.86 \%$ \\
\cline { 2 - 4 } & From 20 to 34 years & 237 & $32.02 \%$ \\
\cline { 2 - 4 } & From 35 to 44 years & 219 & $29.59 \%$ \\
\cline { 2 - 4 } & From 45 to 55 years & 77 & $10.40 \%$ \\
\cline { 2 - 4 } & Greater than 55 years & 60 & $8.10 \%$ \\
\hline \multirow{3}{*}{$\begin{array}{c}\text { Duration } \\
\text { of club } \\
\text { fandom }\end{array}$} & less than 5 years & 189 & $25.54 \%$ \\
\cline { 2 - 4 } & 6 to 10 years & 382 & $51.62 \%$ \\
\cline { 2 - 4 } & 11 to 20 years & 92 & $12.43 \%$ \\
\cline { 2 - 4 } & From 21 to 30 years & 41 & $5.54 \%$ \\
\cline { 2 - 4 } & More than 30 years & 36 & $4.86 \%$ \\
\hline \hline
\end{tabular}

\section{Data collection tools:}

The researcher used the following in research data collection:

- $\quad$ Reviewing researches and scientific references on brands and fans' loyalty in football such as Saad Shalaby, Naji Ismail (2013), Dina Abdel Hakim (2015), Richelieu et al (2011) Bauer et al (2008) ، Maderer et al (2016), Biscaia (2015), Bridgewater (2010), Karjaluoto et al. (2016), that showed the most important axes and dimensions discussed by those researchers, and their most important findings.

- Making a scale developed with closed-answer questions using Likert scale (1
$=$ not effective, $5=$ very powerful effect), and the researcher followed following steps in making it:

Identify axes and phrases:

Through researcher review for Arabic and foreign references related to brands and fans' loyalty in football, the researcher identified two axes:

- First axis: club's variables, included dimensions:

- First dimension: club popularity, included phrases.

- Second dimension: winning and performance onfield, included (7) phrases. 
- Third dimension: players, included (7) phrases.

- Fourth dimension: club management, included (6) phrases.

- Second axis: fan's variables, included dimensions:

- First dimension: Community pride included (3) phrases.

- Second dimension: social aspects, included (4) phrases.

- Third dimension: fans' identity, included (5) phrases.

- Sports Brands' behavioral loyalty scale (Bauer et al. 2008), this scale has been used because of:

- Many researchers in football context used that scale (such: Richelieu et al 2011, Maderer et al. 2016) because of its compatibility with fans and their behavior.

- This scale enjoys high honesty and consistency.

Table (2)

Correlation coefficients between phrases, axis and scale total score $(n=75)$

\begin{tabular}{|c|c|c|c|c|c|c|c|c|c|c|c|}
\hline \multicolumn{12}{|c|}{ First axis: the variables related to club } \\
\hline \multicolumn{3}{|c|}{ First: club popularity } & \multicolumn{3}{|c|}{$\begin{array}{l}\text { Second: Winning and } \\
\text { performance on-field }\end{array}$} & \multicolumn{3}{|c|}{ Third: Players } & \multicolumn{3}{|c|}{ Fourth: club Management } \\
\hline \multirow{2}{*}{ Phrases } & \multicolumn{2}{|c|}{ Link Values } & \multirow{2}{*}{ Phrases } & \multicolumn{2}{|c|}{ Link Values } & \multirow{2}{*}{ Phrases } & \multicolumn{2}{|c|}{ Link Values } & \multirow{2}{*}{ Phrases } & \multicolumn{2}{|c|}{ Link Values } \\
\hline & Axis & questionnaire & & Axis & questionnaire & & Axis & questionnaire & & Axis & questionnaire \\
\hline 1 & $810^{*}$ & $.614 *$ & 1 & .835 & $.667 *$ & 1 & $619 *$ & $.388 *$ & 1 & $858 *$ & $.652 *$ \\
\hline$\overline{2}$ & $.762 *$ & $.592 *$ & 2 & $.822^{*}$ & $.671 *$ & 2 & $.534 *$ & $.438^{*}$ & 2 & $.550 *$ & $.487 *$ \\
\hline 3 & $.694 *$ & $.552 *$ & 3 & $.808^{*}$ & $.650 *$ & 3 & $.531 *$ & $.663^{*}$ & 3 & $.783^{*}$ & $.578 *$ \\
\hline 4 & $.670 *$ & $.445^{*}$ & 4 & $.522^{*}$ & $.360^{*}$ & 4 & $.816^{*}$ & $.458^{*}$ & 4 & $.607^{*}$ & $.529^{*}$ \\
\hline 5 & $.739^{*}$ & $.523^{*}$ & 5 & $.650^{*}$ & $.490^{*}$ & 5 & 0.203 & 0.129 & 5 & $.740 *$ & $.615^{*}$ \\
\hline
\end{tabular}

Assiut Journal For Sport Science Arts
- This scale have been translated from English into Arabic and then translated into English again to ensure language safety.

Pilot Study:

To verify suitability of scale phrases, researcher applied questionnaire (Appindex 1) and scale (Appindex 2) on a sample of (75) Egyptian football fans inside research community and outside basic study sample. The researcher used validity of internal consistency of both scales by finding correlation coefficients between each phrase with axis total score, and scales total score, correlation coefficients between axes, and correlation coefficients between total score of each axis and scale total score.

Internal consistency: 


\section{Follow Table (2) \\ Correlation coefficients between phrases, axis and scale total score $(\mathbf{n}=\mathbf{7 5})$}

\begin{tabular}{|c|c|c|c|c|c|c|c|c|c|c|c|}
\hline \multicolumn{12}{|c|}{ First axis: the variables related to club } \\
\hline \multicolumn{3}{|c|}{ First: club popularity } & \multicolumn{3}{|c|}{$\begin{array}{l}\text { Second: Winning and } \\
\text { performance on-field }\end{array}$} & \multicolumn{3}{|c|}{ Third: Players } & \multicolumn{3}{|c|}{ Fourth: club Management } \\
\hline \multirow{2}{*}{ Phrases } & \multicolumn{2}{|c|}{ Link Values } & \multirow{2}{*}{ Phrases } & \multicolumn{2}{|c|}{ Link Values } & \multirow{2}{*}{ Phrases } & \multicolumn{2}{|c|}{ Link Values } & \multirow{2}{*}{ Phrases } & \multicolumn{2}{|c|}{ Link Values } \\
\hline & Axis & questionnaire & & Axis & questionnaire & & Axis & questionnaire & & Axis & questionnaire \\
\hline 1 & $810^{*}$ & $.614 *$ & 1 & .835 & $.667 *$ & 1 & $619 *$ & $.388 *$ & 1 & $858 *$ & $.652 *$ \\
\hline$\overline{6}$ & 0.106 & 0.092 & $\overline{6}$ & $.759 *$ & $2.630^{*}$ & $\overline{6}$ & $.805 *$ & $.499 *$ & 6 & $.737^{*}$ & $.699 *$ \\
\hline 7 & $.445^{*}$ & $.405^{*}$ & 7 & $.710 *$ & $.622^{*}$ & 7 & $.647^{*}$ & $.388^{*}$ & & & \\
\hline \multicolumn{9}{|c|}{ Second axis: Fan's variables } & \multirow{2}{*}{\multicolumn{3}{|c|}{ Brand's behavioral loyalty scale }} \\
\hline \multicolumn{3}{|c|}{ First: Community pride } & \multicolumn{3}{|c|}{ Second: society aspects } & \multicolumn{3}{|c|}{ Third: fan's identity } & & & \\
\hline \multirow[b]{2}{*}{ Phrases } & \multicolumn{2}{|c|}{ Link Values } & \multirow[b]{2}{*}{ Phrases } & \multicolumn{2}{|c|}{ Link Values } & \multirow[b]{2}{*}{ Phrases } & \multicolumn{2}{|c|}{ Link Values } & \multirow[b]{2}{*}{ Phrases } & \multirow{2}{*}{\multicolumn{2}{|c|}{$\begin{array}{c}\text { Link Values } \\
\text { Axis }\end{array}$}} \\
\hline & Axis & questionnaire & & Axis & questionnaire & & Axis & questionnaire & & & \\
\hline 1 & $.544 *$ & $.364 *$ & 1 & $.878^{*}$ & $.407 *$ & 1 & $.755^{*}$ & $.430^{*}$ & 1 & & $.550 * *$ \\
\hline 2 & $.849^{*}$ & $.314^{*}$ & 2 & $.504 *$ & $.502^{*}$ & 2 & $.711 *$ & $.456^{*}$ & 2 & & $.461 * *$ \\
\hline \multirow[t]{4}{*}{3} & $.850^{*}$ & $.550^{*}$ & 3 & $.325 *$ & $.286^{*}$ & 3 & 0.165 & 0.103 & 3 & & $.649^{* *}$ \\
\hline & & & 4 & $.480 *$ & $.528 *$ & 4 & $.713^{*}$ & $.436^{*}$ & 4 & & $.813^{* *}$ \\
\hline & & & & & & 5 & $.680^{*}$ & $.277^{*}$ & 5 & & $.648^{* * *}$ \\
\hline & & & & & & & & & & & $.738^{* *}$ \\
\hline
\end{tabular}

* Tabular value $(\mathrm{R})$ at significant level $(0.05)=0.198$

As shown in Table (2), there is a statistically significant correlation between phrases with axis total degree and scale total score, where calculated $(R)$ value is greater than tabular value at a significant level (0.05).

There are also no significant differences between numbers

\section{Table (3)}

Correlation coefficients between scale axes $(n=75)$

\begin{tabular}{|c|c|c|c|c|c|c|c|c|c|}
\hline & & \multirow[b]{2}{*}{ axes } & \multicolumn{4}{|c|}{ first axis } & \multicolumn{3}{|c|}{ second axis } \\
\hline & & & $\begin{array}{c}\text { Club } \\
\text { popularity }\end{array}$ & $\begin{array}{c}\text { winning and } \\
\text { performance } \\
\text { on-field }\end{array}$ & players & $\begin{array}{c}\text { Club } \\
\text { management }\end{array}$ & $\begin{array}{c}\text { Community } \\
\text { pride }\end{array}$ & $\begin{array}{c}\text { Social } \\
\text { aspects }\end{array}$ & $\begin{array}{l}\text { Fan's } \\
\text { identity }\end{array}$ \\
\hline 1 & \multirow{4}{*}{$\begin{array}{l}\text { First } \\
\text { axis }\end{array}$} & $\begin{array}{l}\text { Club } \\
\text { Popularity }\end{array}$ & 1 & $.760 *$ & $.385^{*}$ & $.701 *$ & $.289^{*}$ & $.364^{*}$ & $.355^{*}$ \\
\hline 2 & & $\begin{array}{l}\text { winning and } \\
\text { performance } \\
\text { on pitch }\end{array}$ & & 1 & $.499 *$ & $.778^{*}$ & $.323^{*}$ & $.277 *$ & $.280 *$ \\
\hline 3 & & players & & & 1 & $.580^{*}$ & $.282 *$ & $.316^{*}$ & $.256^{*}$ \\
\hline 4 & & $\begin{array}{l}\text { Club } \\
\text { management }\end{array}$ & & & & 1 & $.254^{*}$ & $.276^{*}$ & $.327^{*}$ \\
\hline 1 & \multirow{3}{*}{$\begin{array}{l}\text { Second } \\
\text { axis }\end{array}$} & $\begin{array}{l}\text { Community } \\
\text { pride }\end{array}$ & & & & & 1 & $.256^{*}$ & $.521^{*}$ \\
\hline 2 & & $\begin{array}{l}\text { Social } \\
\text { aspects }\end{array}$ & & & & & & 1 & $.281^{*}$ \\
\hline 3 & & $\begin{array}{l}\text { Fan's } \\
\text { identity }\end{array}$ & & & & & & & 1 \\
\hline
\end{tabular}

* Tabular value $(\mathrm{R})$ at significant level $(0.05)=0.198$

Assiut Journal For Sport Science Arts popularity, players, fan's identity) respectively with axis total score and with scale total score, where $(\mathrm{R})$ value is less than tabular value at a significant level (0.05), so the researcher deleted these phrases.

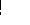


Table (3) shows that there is a statistically significant correlation between survey axes, ranging from
(.254) and (.778), where (R) value is greater than its tabular value at a significant level $(0.05)$.

\section{Table (4)}

correlation coefficients between axes and scale $(n=75)$

\begin{tabular}{|c|c|c|c|}
\hline & & axes & correlation values with scale \\
\hline 1 & \multirow{4}{*}{$\begin{array}{l}\text { First } \\
\text { axis }\end{array}$} & Club Popularity & $.797 *$ \\
\hline 2 & & winning and performance on pitch & $.806^{*}$ \\
\hline 3 & & players & $.703 *$ \\
\hline 4 & & Club management & $.829 *$ \\
\hline 1 & \multirow{3}{*}{$\begin{array}{l}\text { Second } \\
\text { axis }\end{array}$} & Community pride & $.517^{*}$ \\
\hline 2 & & Social aspects & $.600 *$ \\
\hline 3 & & Fan's identity & $.553^{*}$ \\
\hline
\end{tabular}

* Tabular value $(\mathrm{R})$ at significant level $(0.05)=0.198$

As shown in Table (4), there is a statistically significant correlation between total score of each axis with scale total score, ranging between (0.517) and (0.829), where calculated $(R)$ value is greater than its tabular value at a significant level (0.05).

\section{Second:} coefficient of stability:

The researcher used alpha Kronbach coefficient and internal consistency to calculate coefficient of stability of axes and scale, as well as calculation of scale stability coefficient, by applying on a sample of (75) Egyptian football clubs' fans.

Table (5)

stability values of axes, scale $(n=75)$

\begin{tabular}{|c|c|c|c|}
\hline & \multicolumn{2}{|r|}{ axes } & alpha Kronbach values for stability \\
\hline 1 & \multirow{4}{*}{$\begin{array}{l}\text { First } \\
\text { axis }\end{array}$} & Club Popularity & $0.709 *$ \\
\hline 2 & & winning and performance on pitch & $0.853 *$ \\
\hline 3 & & players & $0.751 *$ \\
\hline 4 & & Club management & $0.803^{*}$ \\
\hline 5 & \multirow{3}{*}{$\begin{array}{l}\text { Second } \\
\text { axis }\end{array}$} & Community pride & $0.821 *$ \\
\hline 6 & & Social aspects & $0.756^{*}$ \\
\hline 7 & & Fan's identity & $0.767 *$ \\
\hline \multicolumn{3}{|c|}{ Total Scale } & $0.882 *$ \\
\hline \multicolumn{3}{|c|}{ sports Brand's behavioral loyalty scale } & $0.712 *$ \\
\hline
\end{tabular}

* High values

Assiut Journal For Sport Science Arts 
Table (5) shows stability of axes and scale, where stability coefficient of scale axes in Alpha Kronbach was (0.751), (0.853), while first scale stability coefficient of was (0.882), second scale stability coefficient was (0.712). Internal consistency of all axes was significant, indicating high stability coefficient of scale axes.

\section{Basic Study:}

After conducting scientific transactions of validity and stability, researcher applied the scale in its final form (Appendix $3 \& 4$ ) to basic sample of (740) fans in the period from $(10 / 8 / 2016)$ to (30/8/2016), and after application, data was collected, organized, to perform appropriate statistical treatments.

Results' presentation and discussion:

First: Results' Presentation and discussion of first hypothesis: There is a positive correlation between club's variables (club popularity, wining and performance on pitch, players, club management) and loyalty to brand.

Table (6)

Relationship between club's variables (club popularity, wining and performance on pitch, players, club management) and loyalty to brand $(n=740)$

\begin{tabular}{l|l|c|c|c|c|c}
\hline \hline \multicolumn{2}{c|}{ axes } & $\begin{array}{c}\text { Club } \\
\text { popularity }\end{array}$ & $\begin{array}{c}\text { winning and } \\
\text { performance } \\
\text { on pitch }\end{array}$ & players & $\begin{array}{c}\text { Club } \\
\text { management }\end{array}$ & $\begin{array}{c}\text { Community } \\
\text { pride }\end{array}$ \\
\hline \hline \multirow{2}{*}{ Club } & $\begin{array}{l}\text { Club } \\
\text { Popularity }\end{array}$ & 1 & $.649 *$ & $.311^{*}$ & $.524 *$ & $.466^{*}$ \\
\cline { 2 - 7 } variables & $\begin{array}{l}\text { winning and } \\
\text { performance } \\
\text { on pitch }\end{array}$ & & 1 & $.389 *$ & $.631 *$ & $.409 *$ \\
\cline { 2 - 7 } & players & & & 1 & $.479 *$ & $.362^{*}$ \\
\cline { 2 - 7 } & $\begin{array}{l}\text { Club } \\
\text { management }\end{array}$ & & & & 1 & $.360^{*}$ \\
\hline \multicolumn{2}{l}{ loyalty to brand } & & & & & 1 \\
\hline \hline
\end{tabular}

* Tabular value $(\mathrm{R})$ at a significant level $(0.05)=0.022$

Table (6) shows that all correlation coefficients between independent variables of first axis (club popularity, pitch, players, club management) and dependent variable (loyalty to brand) were statistically significant at wining and performance on (0.05). This indicated positive 
correlation relationship between each variable from independent and dependent variables. The researcher attributes this result to mixed effect between independent variable "club" (under research) and dependent variables "loyalty to football clubs' brands", this is due to interaction between these variables and their role in explaining loyalty to brand. This is agrees with Richelieu et al (2011), which referred to many motivating factors in building brand identity and loyalty such as club history, club performance on pitch, style of play, foreign stars, local stars, team manager, club logo, public relations and community involvement.(15)

This is confirmed by Maderer et al (2016) that dimensions as team history, organizational factors, team success, principal coach, management, and stadium, should be taken into consideration when talking about team's brand positioning (13).

Thus, validity of first hypothesis can be accepted: There is a positive correlation between club's variables (club popularity, wining and performance on pitch, players, club management) and loyalty to brand.

\section{Second: Results' Presentation} and discussion of second hypothesis: There is an influence of club's variables (club popularity, wining and performance on pitch, players, club management) combined on loyalty to brand.

Table (7)

Results of Multiple linear regression analysis of club's independent variables effect on dependent variable "loyalty to brand"

\begin{tabular}{|c|c|c|c|c|c|c|c|c|}
\hline \multicolumn{2}{|c|}{$\begin{array}{l}\text { Independent } \\
\text { Variables }\end{array}$} & $\mathrm{R}^{2}$ & $\begin{array}{l}\text { Regression } \\
\text { coefficient }\end{array}$ & $\begin{array}{c}\text { Standard } \\
\text { Error }\end{array}$ & B & $\begin{array}{c}\mathrm{T} \\
\text { Value }\end{array}$ & $\begin{array}{c}\text { Probability } \\
\text { Value sig }\end{array}$ & ranking \\
\hline \multicolumn{2}{|c|}{ Club's Variables } & .276 & 4.755 & 1.075 & & 4.423 & .000 & \\
\hline 1 & $\begin{array}{l}\text { club } \\
\text { popularity }\end{array}$ & .217 & .384 & .051 & .318 & 7.567 & .000 & 1 \\
\hline 2 & $\begin{array}{l}\text { wining and } \\
\text { performance } \\
\text { on pitch }\end{array}$ & .167 & .212 & .037 & .205 & 5.686 & .000 & 2 \\
\hline 3 & players & .131 & .106 & .048 & .102 & 2.192 & .029 & 3 \\
\hline
\end{tabular}


Follow Table (7)

Results of Multiple linear regression analysis of club's independent variables effect on dependent variable "loyalty to brand"

\begin{tabular}{|c|c|c|c|c|c|c|c|c|}
\hline \multicolumn{2}{|c|}{$\begin{array}{l}\text { Independent } \\
\text { Variables }\end{array}$} & $\mathrm{R}^{2}$ & $\begin{array}{l}\text { Regression } \\
\text { coefficient }\end{array}$ & $\begin{array}{l}\text { Standard } \\
\text { Error }\end{array}$ & B & $\begin{array}{c}\mathrm{T} \\
\text { Value }\end{array}$ & $\begin{array}{c}\text { Probability } \\
\text { Value sig }\end{array}$ & ranking \\
\hline 4 & $\begin{array}{l}\text { club } \\
\text { managemen }\end{array}$ & .129 & .032 & .042 & .033 & .761 & .447 & 4 \\
\hline \multicolumn{9}{|c|}{ Analysis of variance ANOVA } \\
\hline 1 & $\begin{array}{l}\text { Test } \\
\text { Value } \\
\text { (F) }\end{array}$ & \multicolumn{2}{|c|}{$\begin{array}{l}\text { Value of the } \\
\text { interpretation } \\
\text { coefficient } \mathrm{R}^{2}\end{array}$} & \multicolumn{3}{|c|}{ Significance } & \multicolumn{2}{|c|}{$\begin{array}{c}\text { probability value of } \\
\text { interpretation } \\
\text { coefficient }\end{array}$} \\
\hline 2 & 69.887 & \multicolumn{2}{|c|}{.276} & \multicolumn{3}{|c|}{0.000} & \multicolumn{2}{|c|}{0.000} \\
\hline
\end{tabular}

Table (7) shows that calculated " $F$ " value is equal to 69.887 and is statistically significant at level 0.05 , where probability value is 0.00 , which is less than 0.05 level. This indicates that there is a statistically significant effect between club's variables (club popularity, wining and performance on pitch, players, club management) and loyalty to brand. This is confirmed by calculated value "T" and significance level of (000), which is lower than research default level (0.05).

There is also a statistically significant relationship between club's variables and loyalty to brand, where value of interpretation coefficient $\mathrm{R}^{2}$ is 0.276 . This means that club's variables contribute $(27.6 \%)$ to loyalty to sports clubs' brands assuming stability of other factors, and this effect is significant at (0.05).

Club's popularity came in first ranking as the club's most influential variable in loyalty to brand with a regression coefficient 0.384 . The coefficient of interpretation $\mathrm{R}^{2} \quad$ (0.217), means that club's popularity variable contributes with $(21.7 \%)$ in loyalty to football clubs' brands, and the researcher attributes this result that most community members have a desire to associate with football club's identity. Because this association occurs at a relatively small age, therefore, the most important criteria of this association is club's popularity and fans' numbers. It can be said that 
there is a positive relationship between club's popularity and fan's identity and their ability to express this association in society.

Wining and performance on pitch came in second ranking, with regression coefficient (0.212), value of interpretation coefficient $\mathrm{R}^{2}$ (0.167), which means that wining and performance on pitch contributes with (16.7\%) in loyalty to sports clubs' brands. In this regard Bridgewater (2010) points out that in case of football brands, brands' performance is clearly related to their clubs' performance on pitch, where any brand in football world can develop its popularity and raise awareness of it, if team was successful and member in a successful league that raise large television coverage. (7:163)

This agrees with Szymanski's (2010), that fans are very sensitive to their teams' success, which means that fans are likely to stop watching matches and buying merchandise, even if loyal fans remained loyal to their teams, they have to keep in mind that there are new fans joining fans' lists every new season.(17)
In this regard, Richelieu et al (2011) confirms that clubs must shed light on success factors on pitch in order to properly manage team's brand.(15)

Players' variable came in third ranking with regression coefficient (0.106), interpretation coefficient value $\mathrm{R}^{2}$ was (0.131), meaning that players' variable contributed $13.1 \%$ to loyalty of sports clubs' brands.

The researcher attributes this result to large number of fans who care about aesthetics and players' skills, even when results are bad, we find that fans are very optimistic about those players. This agree with Hoegele et al. (2014), that players contribute in attracting new fans, while preventing fans from being attracted to rival teams. There is also a positive relationship between star players and not changing loyalty to other teams.(10)

Kirk Wakefield (2007) points out that some teams' marketers shed light on a certain player in their advertising and promotional campaigns as one of rising players, so fans continue loyal to their teams $(3: 28)$. 
Club management variable came in final ranking with a regression coefficient (0.032), and value of interpretation coefficient $R^{2}$ (0.129), meaning that club management variable contributes $(12.9 \%)$ in loyalty to sports clubs' brands of value overall effect for club's variables, with assuming stability of other factors.

This agrees with Bridgewater (2010) that fans are interested in their clubs' financial stability, where they prefer to provide funds to achieve more success and to plan for future growth. They also consider it very important that clubs have a "good" and stable management. (7:29)

Thus, validity of second hypothesis can be accepted: club's variables (club's popularity, winning and performance on pitch, players, club management) all influence on loyalty to brand.

Third: Results' Presentation and discussion of third hypothesis: There is a positive correlation between fan's variables (community pride, social aspects, fan's identity) and loyalty to brand.

Table (8)

Relationship between fan's variables (community pride, social aspects, fan's Identity) and loyalty to brand $(n=740)$

\begin{tabular}{l|l|c|c|c|c}
\hline \hline \multicolumn{2}{c|}{ axes } & $\begin{array}{c}\text { Community } \\
\text { Pride }\end{array}$ & $\begin{array}{c}\text { Community } \\
\text { Social } \\
\text { aspects }\end{array}$ & $\begin{array}{c}\text { Fan's } \\
\text { Identity }\end{array}$ & $\begin{array}{c}\text { loyalty } \\
\text { to } \\
\text { brand }\end{array}$ \\
\hline \hline \multirow{2}{*}{ Fan's } & $\begin{array}{l}\text { Community } \\
\text { Pride }\end{array}$ & 1 & $.514^{*}$ & $.556^{*}$ & $.544^{*}$ \\
\cline { 2 - 6 } variables & $\begin{array}{l}\text { Community } \\
\text { Social } \\
\text { aspects }\end{array}$ & & 1 & $.484^{*}$ & $.592^{*}$ \\
\cline { 2 - 6 } & $\begin{array}{l}\text { Fan's } \\
\text { Identity }\end{array}$ & & & 1 & $.611^{*}$ \\
\hline loyalty to brand & & & & 1 \\
\hline \hline
\end{tabular}

*Tabular value $(\mathrm{R}) \mathrm{t}$ a significant level $(0.05)=0.022$

Table (8) shows that all social aspects, fan's identity) correlation coefficients between independent variables and dependent variable (loyalty of second axis (social pride, to brand) were statistically significant at (0.05). This 
indicates a positive correlation between each independent and dependent variable. The researcher attributes this result to mixed effect between fan's independent variables (under research) and dependent variable "loyalty to brand", this is due to interaction between these variables and their role in loyalty interpretation to football clubs' brands.

This agrees with Kargaluoto et al (2016), that brand personality is a strong driver of identification among new fans, where results confirmed importance of sports brands in fans' identification with teams and their loyalty to them.(11), Kirk Wakefield (2007) points out that there are

\section{Table (9)}

Results of Multiple linear regression analysis of fan's independent variables effect on dependent variable "loyalty to brand"

\begin{tabular}{|c|c|c|c|c|c|c|c|c|}
\hline \multicolumn{2}{|c|}{$\begin{array}{c}\text { Independent } \\
\text { Variables }\end{array}$} & $\mathbf{R}^{2}$ & $\begin{array}{l}\text { Regression } \\
\text { coefficient }\end{array}$ & $\begin{array}{c}\text { Standard } \\
\text { Error }\end{array}$ & B & $\begin{array}{c}\mathbf{T} \\
\text { Value }\end{array}$ & $\begin{array}{c}\text { Probability } \\
\text { Value sig }\end{array}$ & ranking \\
\hline \multicolumn{2}{|c|}{ Fan's Variables } & .508 & 4.077 & .699 & & 5.830 & 0.000 & \\
\hline 1 & $\begin{array}{l}\text { Community } \\
\text { Pride }\end{array}$ & .295 & .327 & .060 & .179 & 5.414 & 0.000 & 3 \\
\hline 2 & $\begin{array}{l}\text { Social } \\
\text { aspects }\end{array}$ & .350 & .448 & .047 & .330 & 10.516 & 0.000 & 2 \\
\hline 3 & $\begin{array}{l}\text { Fan's } \\
\text { identity }\end{array}$ & .373 & .495 & .041 & .352 & 10.869 & 0.000 & 1 \\
\hline \multicolumn{9}{|c|}{ Analysis of variance ANOVA } \\
\hline 1 & $\begin{array}{l}\text { Test } \\
\text { Value } \\
(\mathrm{F})\end{array}$ & \multicolumn{2}{|c|}{$\begin{array}{l}\text { Value of the } \\
\text { interpretation } \\
\text { coefficient } \mathrm{R}^{2}\end{array}$} & \multicolumn{3}{|c|}{ Significance } & \multicolumn{2}{|c|}{$\begin{array}{l}\text { probability value of } \\
\text { interpretation coefficient }\end{array}$} \\
\hline 2 & 253.068 & \multicolumn{2}{|c|}{.508} & \multicolumn{2}{|c|}{0.000} & & \multicolumn{2}{|l|}{0.000} \\
\hline
\end{tabular}

Assiut Journal For Sport Science Arts 
Table (9) shows that calculated "F" value is 253.068, it is statistically significant at level 0.05 , where probability value is 0.00 , below 0.05 . This indicates that there is a statistically significant effect between fan's variables (community pride, social aspects, and fan's identity) and loyalty to brand. This is confirmed by calculated value "T" and its significance level (000), which is lower than research default level (0.05).

It is also evident that there is a statistically significant relationship between club's variables and loyalty to brand, where value of interpretation coefficient $R^{2}$ is (0.508), which means that fan's variables contribute with $50.8 \%$ to loyalty to sports clubs' brands, assuming stability of other factors, this effect is significant (0.05).

Fan's identity came in first ranking as the most affective variables in loyalty to brand, with a regression coefficient (0.495), and value of interpretation coefficient $\mathrm{R}^{2}$ (0.373), which means that fan's identity variable contributes $(37.3 \%)$ in loyalty to sports club brand.
Bridgewater (2010) points out that loyalty and identification are also one of the themes that fascinate fans themselves, where they spend huge monies in their lives supporting football by trying to show their "loyal" attitude in front of fans, either of their clubs or other clubs. (7:74)

Social aspects came in second ranking by a regression coefficient (0.448), and interpretation coefficient $\mathrm{R}^{2}$ (0.350). This means that social aspects variable contributes with $35 \%$ to loyalty to sports clubs' brands.

This agrees with Biscaia et al. (2015), which indicated that fans' chance to interact with friends and other fans during football events has a great importance and so social interaction is often highlighted as a benefit of football consumption and this helps to understand brand position in football.(5)

Fetchko et al (2013) points out that sport attracts attention through other social technique "belonging to group" that includes people who want to communicate with others who share passion for any team or sports personality, and that motivation enables them to 
achieve their goal of becoming "fans". (8:30)

Community pride came in third ranking, with regression coefficient by (0.327) and interpretation coefficient $R^{2}$ (0.295). This means that community pride variable contributes with $(29.5 \%)$ to loyalty of sports clubs'.

This agrees with Bridgewater (2010) that community pride is one of the factors that contribute to fans supporting local teams, or any teams associated with their family roots, or where they lived at some point in their lives. (7:63)

Thus, validity of fourth hypothesis can be accepted: fans' variables (community pride, social aspects, and fan's identity) all influence on loyalty to brand.

\section{Conclusions:}

- There is a positive correlation between club's variables (club popularity, wining and performance onfield, players, club management) and loyalty to brand.

- Club's variables influence (club popularity, wining and performance onfield, players, club management) combined on loyalty to brand.

- There is a positive correlation between fan's variables (community pride, social aspects, and fan's identity) and loyalty to brand.

- Fans' variables influence (community pride, social aspects, and fan's identity) combined on loyalty to brand.

- $\quad$ Research found that fans' identity is the most influential of fans' variables in loyalty phenomenon to football clubs' brands.

- Research found that club's popularity is the most influential of club's variables in loyalty phenomenon to football clubs' brands.

- fan's variables contribute by $50.8 \%$ in interpretation of brand loyalty, while club's variables contribute by $27.6 \%$, with assuming stability of other factors.

\section{Recommendations:}

- Football clubs' need to pay attention to variables that affect fans' loyalty to brand to manage it properly.

- Football clubs' need to determine brands' value in sports market continuously and benefit from this value in enhancing fans' loyalty. 
- $\quad$ Sports marketers should provide opportunities for fans to participate in social events before and after matches to develop positive feelings towards the club and promote loyalty to brand.

- Football clubs should conduct their own research to see how fans look at their brands and know if there is a gap between organization and fans' expectations

- Sports marketers must take brand as a cornerstone of marketing initiatives for football clubs.

- Football clubs' need to create loyalty programs for loyal fans to enhance their loyalty to club's brand, such as:

- Permanent

communication with fans

- Enhancing social experiences of fans

- Enable communication with fans

- Offering rewards and motivations to loyal fans.

\section{References:}

1- Dina Abdel Hakim (2015): The Role of Corporate Sponsorship for Sports Events in Promoting Brand's Value, Unpublished Master Thesis, Faculty of Media, Cairo University.

\section{2- Saad Ahmed Shalaby, Naji} Ismail Hamid. (2013): Managing Agreements of licensing Brands' Programs in Egyptian \& Emirates' football industry "A Comparative Study", The Egyptian Journal of Commercial Studies, Volume 37, No. 3.

3- Kirk Wakefield (2007). Marketing of sports teams, translation Dar Al-Farouk, Giza, Egypt.

4- Bauer, H. H., StokburgerSauer, N.E. \& Exler, S. (2008). Brand image and fan loyalty in professional team sport: a refined model and empirical assessment", Journal of Sport Management, Vol. 22 No. 2, pp. 205-226.

5- Biscaia, R., Ross, S., Yoshida, M., Correia, A., Rosado, A \& Maroco, J. (2015). Investigating the role of fan club membership on perceptions of team brand equity in football, Sport Management Association of Australia and New Zealand, Elsevier Ltd.

6- Boyle, B. A., \& Magnusson, P. (2007). Social identity and brand equity formation: A comparative study of collegiate sports fans. Journal of Sport Management, 21(4), 497-520. 
7- Brand Finance. (2011). The Annual Report on Europe's most valuable Football Brands, September.

8- Bridgewater, S. (2010). Football Brands, Palgrave, Macmillan, London, UK.

9- Fetchko, M. Roy, D. \& Clow, K. (2013). Sports Marketing, Prentice Hall, U.S.A.

10- Hoegele., D, Schmidt, S., \& Benno., T. (2014). Superstars and disloyal football fans: factors influencing attraction to competition, Sport, Business and Management: An International Journal, Vol. 4 Issue: 4, pp.298-316

11- karjaluoto., H. Munnukka, J.\& Salmi, M. (2016). How do brand personality, identification, and relationship length drive loyalty in sports?", Journal of Service Theory and Practice, Vol. 26 Issue: 1,pp.50-71.

12- Koo, G.-Y. \& Hardin, R. (2008). Difference in interrelationship between spectators' motives and behavioral intentions based on emotional attachment, Sport Marketing Quarterly, 17(1), 30-43

13- Maderer., D. Holtbruegge., D.\& Woodland., R. (2016). The impact of brand associations on brand loyalty in the football industry: A comparison of fans from developed and emerging football markets, Sport, Business and Management: An International Journal, Vol. 6 Issue: 5, pp.499-519,

14- Martin, C.A. (2013). Investigating National Football League (NFL) fan loyalty, Journal of Marketing Development and Competitiveness, Vol. 7 No. 1, pp. 42-53.

15- Richelieu., A. Pawlowski .,T., \& Breuer., C.(2011). Football brand management: Minor league versus Champions League, Journal of Sponsorship Vol. 4. No. 2. 178 178-189.

16- Ross, S.D., Russell, K.C. \& Bang, H. (2008). An empirical assessment of spectator-based brand equity, Journal of Sport Management, Vol. 22 No. 3, pp. 322-337.

17- Szymanski, S.(2010) .Commercial Football and the Economic Cycle, In: Butenko, S., Lafuente, J., \& Pardalos. P. Optimal Strategies in Sports Economics and Management, Springer, London.

18- Theodorakis, N. D., Alexandris, K., Tsigilis, N., \& Karvounis, S. (2013). Predicting spectators' behavioural intentions in professional football: The role of satisfaction and service quality. Sport Management Review, 16(1), 85-96. 